\title{
Finite Element Analysis of MW Permanent Magnet Synchronous Generator
}

\author{
Chengxin Xu \\ Chengdu University of Information Technology, Chengdu, 610225, China \\ Email:xcx@cuit.edu.cn
}

Keywords: finite element; permanent magnet synchronous generator; static magnetic field

\begin{abstract}
Basic theory of finite element analysis of electromagnetic field was expounded in this paper. Boundary conditions, boundary value problem and condition variety problem were included. After above work, boundary condition selection and model structure were processed. After meshing, static magnetic field distribution were calculated. And distribution of magnetic line and gap flux density were obtained.
\end{abstract}

\section{Overview}

Magnetic circuit of permanent magnet synchronous generator has many types. And with the development of technique, it will be richer. Direct numerical analysis of electromagnetic field is necessary for improving accuracy in new type design. Moreover, the number of permanent magnet motor electromagnetic processes with distinct characteristics and some specific issues, such as the optimization of the structure and size of the pole, permanent partial loss of field problems and permanent magnet synchronous motor starting process, also requires the use of numerical electromagnetic field to perform quantitative analysis ${ }^{[1]}$. With the rapid development of computer technology, the current permanent magnet motor design methods rely on electromagnetic numerical method more and more.

\section{Finite Element Theory of Electromagnetic Field}

\subsection{Boundary Condition}

Currently, determine solution problem which only has boundary condition and has no initial condition is the main study direction in generator electromagnetic problem ${ }^{[2]}$.

Boundary conditions are usually three forms:

(1) The physical conditions of the predetermined physical boundary values u on the boundary of $\Gamma$

$$
\left.u\right|_{\Gamma}=f_{1}(\Gamma)
$$

This is called the first boundary condition. When the physical value of zero on the boundary, known as the first class of homogeneous boundary conditions.

(2) Physical conditions on the boundary defines the value of the derivative u physical law on border

$$
\left.\frac{\partial u}{\partial n}\right|_{\Gamma}=f_{2}(\Gamma)
$$

This is called the second boundary condition. $U$ is zero when the derivative law, known as the second class of homogeneous boundary conditions.

(3) Physical conditions on the boundary defines a linear relationship between the derivative and the law on physical u border

$$
\left.\left(\eta u+\beta \frac{\partial u}{\partial n}\right)\right|_{\Gamma}=f_{3}(\Gamma)
$$


Wherein: $\eta, \beta$ - constant, it is called third boundary condition.

The study of the magnetic field problem, in most cases first class and second class of boundary conditions are used. Their division is relevant with selection of solving function ${ }^{[3]}$.

\subsection{Boundary Question and Condition Variety Question}

Electromagnetic field generally would not consider the impact of displacement currents what are up the field ${ }^{[4]}$. Analysis of the motor up to a vertical plane parallel to the axis of the motor field, then only the component of the current density and magnetic vector potential in the z-axis direction. For steady-state conditions, electromagnetic field $\Omega$ problems on the plane can be expressed as a boundary value problem

$$
\left\{\begin{array}{c}
\Omega: \frac{\partial}{\partial n}\left(v \frac{\partial A}{\partial x}\right)+\frac{\partial}{\partial y}\left(v \frac{\partial A}{\partial y}\right)=-J_{z} \\
\Gamma_{1}: A=A_{0} \\
\Gamma_{2}: v \frac{\partial A}{\partial n}=-H_{t}
\end{array}\right.
$$

Wherein: $v-$ magnetic reluctivity, $v=1 / \mu, \mu$ is magnetic conductivity;

$\mathrm{A}$ - Magnetic vector potential, because only the z-axis component $\mathrm{A}$, it can be written in the form of scalar;

$\mathrm{J}_{\mathrm{Z}}$ - Source current density;

$\mathrm{H}_{\mathrm{T}}$ - The tangential component of the magnetic field strength;

$\Gamma_{1}$ - The first boundary;

$\Gamma_{2}$ - The second boundary.

Then all the magnetic field lines are in the xy plane. And the $\mathrm{x}$-axis and magnetic field components only have the $y$-axis direction. Their expressions were:

$$
B_{x}=\frac{\partial A}{\partial y} \quad B_{y}=-\frac{\partial A}{\partial x}
$$

In determining the boundary condition on the boundary often used flux density Bt and the magnetic flux density Bn tangential direction normal to the direction generally tangential direction $\mathrm{t}$ of the $n$ predetermined rotated counterclockwise by $90^{\circ}$ in the positive direction of the outer normal n, In this case:

$$
B_{n}=\frac{\partial A}{\partial t} \quad B_{t}=-\frac{\partial A}{\partial n}
$$

Expression (6) is equivalent to variety question:

$$
\begin{gathered}
\left\{\begin{array}{c}
W(A)=\iint_{\Omega}\left(\int_{0}^{B} v B d B-J_{Z} A\right) d x d y-\int_{\Gamma_{2}}\left(-H_{t}\right) A d l=\min \\
\Gamma_{1}: A=A_{0}
\end{array}\right. \\
\text { Wherein: }
\end{gathered}
$$

\section{Determinations of Boundary Conditions}

Finite element method for solving electromagnetic field, will solve the region is conducive to solving narrow range, typically the outer surface of the motor as the boundary surface, which belongs to impose the boundary surface ${ }^{[5]}$. Since the magnetic permeability of ferromagnetic materials is much larger than the air permeability of this approximation can be applied to 
engineering problems. Generally considered the outer surface of the motor along the magnetic field lines are closed, this border belongs to the first class of homogeneous boundary. In many cases the outer surface of the motor shaft is also taken as the first class of homogeneous boundary. Solving regional boundary conditions of megawatt permanent magnet synchronous generator is

$$
\left.A\right|_{A B C D}=0
$$

If the stator core is so saturated that external magnetic flux leakage is necessary to be considered, this artificial boundary should expand outwards appropriate. Outward expansion of the scope of the very strict limits, because this part of the magnetic field is weak, the decay quickly.

\section{Finite Element Analysis of Model}

\subsection{Model Construct}

In Maxwell, any entity is composed of the following basic elements: the key point, line, surface, body. The relationship between these four basic elements can be described as follows: the key points can be connected into the line by line to face surrounded by plane after rotating, dragging and other operations to produce the body. Geometric model can be established in the Define Model Maxwell to establish a direct, or you can draw in AutoCAD and then imported into Maxwell in the past. In order to ensure the accuracy of electromagnetic field analysis, the geometric model of the motor design is based on the actual size of the establishment of the 1:1 ratio. Structure described herein megawatt permanent magnet synchronous generator is symmetrical, taking into account the relatively large size of the structure, where only create two-dimensional model for analysis, the following diagram is built according to the motor program to determine the basic parameters of the selected motor geometry.

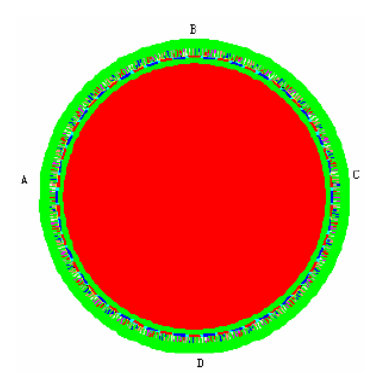

Fig.1 Model of MW Permanent Magnet Synchronous Generator

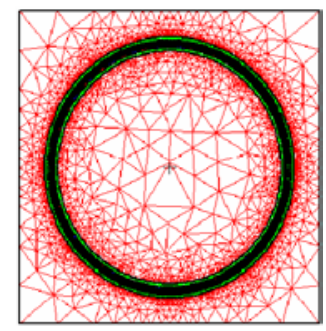

Fig.2 Meshing of Generator

\subsection{Meshing}

In Maxwell, there are powerful meshing tool that can automatically mesh the model, using adaptive mesh while meshing analysis, the system performs an iterative solution of the region's largest mesh refinement error exists, get the higher mesh density, thereby generating a more accurate solution. In each iteration, calculating the total energy of the system. As the percentage of energy to solve the errors caused, thus solving checks to determine whether the percentage of energy has ended, or if the percentage change in the percentage of error and the two most recent error reaches a specified value. In the process of computing software, the software will automatically increase the number of mesh according to a calculation error at each step, until the 
next calculation results meet the design requirements. Figure 2 is a diagram mesh megawatt permanent magnet synchronous generator wind model.

\subsection{Static Magnetic Field Analysis of Model}

Analysis in this paper, the static magnetic field was solved after eight complete adaptive solution processes. Ultimately solving information and data convergence are as shown in Figure 3, it can be seen from the figure, the last divided 125,128 solving triangular elements.

Maxwell 2D/3D provides a powerful post-processing functions, in this part of the user can visually see the distribution of the electromagnetic field inside the motor. This paper analyzes the post-treatment, given the magnetic field lines megawatt permanent magnet synchronous generator wind, air-gap flux density distribution. Since the model of the motor is relatively large, in order to see the distribution of magnetic field lines, Fig 4 shows the distribution of the magnetic line portion area. Figure 5 is a pole next to the distribution of flux density.

\begin{tabular}{|c|c|c|c|c|c|}
\hline \multicolumn{6}{|c|}{ CONVERGEMCE DLTA } \\
\hline Number of passes: & Pass & Triangles & Total Enecgy(J) & EnergY EIroI (쉬| & Kag Force (II) \\
\hline Conpleted 8 & 1 & 6410 & $3.58321 E+004$ & 15.2064 & 2. $39841 E+000$ \\
\hline Renoining & 2 & 9372 & $3.27014 \mathrm{E}+004$ & 2.0196 & $7.35739 \mathrm{E}+002$ \\
\hline 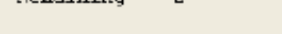 & 3 & $14 \mathrm{~b} \angle \mathrm{U}$ & $3.1 / 44 \angle E+$ UU 4 & บ. $4 \Delta b \mathrm{~b}$ & 1. UbY $\triangle \triangle \mathrm{L}+\mathrm{U} \mathrm{U} /$ \\
\hline & 4 & 21620 & 3. $13 \mathrm{a}+\mathrm{BE}+004$ & 0.1913 & a. $17674 \mathrm{E}+001$ \\
\hline Convergence eciteria: & 5 & 33814 & 3. $13912 \mathrm{E}+004$ & 0.1051 & $2.2569 .3 \mathrm{E}+002$ \\
\hline larget error: U. $1 \angle \mathrm{x}$ & 0 & 53500 & $3.12970 \mathbb{E}+004$ & 0.0520 & $1.55731 \mathrm{E}+002$ \\
\hline Energy Error: 0.0117\% & 7 & 84280 & $3.11800 \mathrm{E}+004$ & 0.0233 & $5.92564 \mathrm{E}+002$ \\
\hline Delta Enecgy: 0.155 s & 8 & 125126 & 3. $11317 \mathrm{E}+004$ & 0.0117 & $3.75676 \mathrm{E}+002$ \\
\hline
\end{tabular}

Fig.3 the convergence of solving data

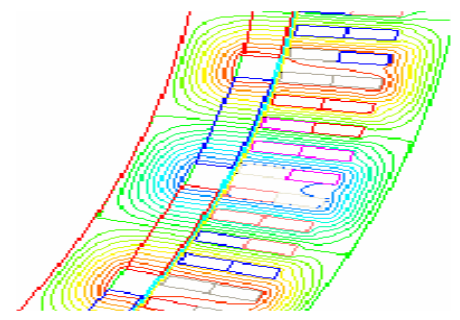

Fig.4 Distribution of Magnetic Line

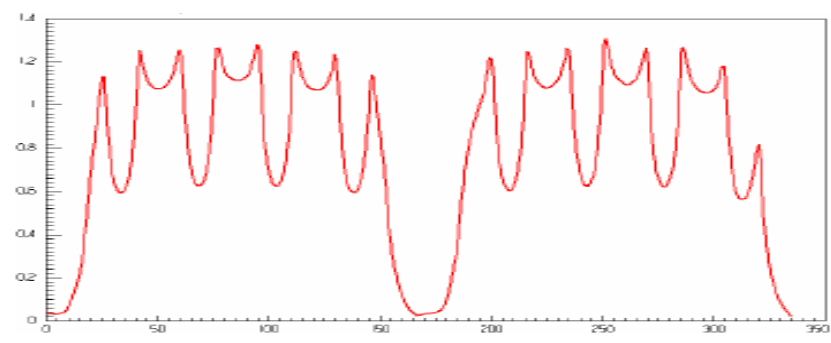

Fig.5 Gap Magnetic Flux Distribution

\section{Conclusions}

Finite element analysis of magnetic field and mathematic calculation of permanent magnet synchronous generator were expounded in this paper. Maxwell 2D of ANSOFT is adopted to analyze the performance of the generator. After modeling, the static magnetic field and no-load magnetic field were analyzed. Finally, calculation method of some parameters of generator with analysis result was introduced. Based on the method, the parameters were calculated and their accuracy was improved.

\section{References}

[1] Global Wind Energy Council (GWEC).Global wind 2012 report. 2013 
[2] L. Soderlund,J-T. Eriksson. A permanent-magnet generator for wind power applications. IEEE Transactions on Magnetics. 1996

[3] Chalmers B.J,, Spooner E. An axial flux permanent-magnet generator for a gearless wind energy system. IEEE Transactions on Energy Conversion. 1999

[4] H Li, Z Chen. Overview of different wind generator systems and their comparisons. IET Renew Power Gener. 2008

[5] G. Shrestha, D. Bang, H. Polinder, etal. Review of generator systems for largewind turbines. Proceedings of EWEC, Brussels Belgium. 2008 